Palavras chave:

Restrições de adjacência Restrições ambientais Florestas equiâneas

Histórico: Recebido 13/04/2011 Aceito 29/04/2014

Keywords: Adjacency constraints Environmental influences Even-aged forest

Correspondência: danielhbbinoti@gmail.com

DOI: |0.1590/0 |04776020|42004| |83
Daniel Henrique Breda Binoti', Mayra Luiza Marques da Silva Binoti', Helio Garcia Leite², José Marinaldo Gleriani ${ }^{2}$, Márcio Leles Romarco de Oliveira', João Carlos Chagas Campos², Agostinho Lopes de Souza², Carlos Antonio Álvares Soares Ribeiro²

\section{REGULAÇÃO DE FLORESTAS EQUIÂNEAS COM RESTRIÇÃO DE CLASSES DE IDADE POR COMPARTIMENTO}

RESUMO: Conduziu-se este estudo, com o objetivo de apresentar a formulação de modelos alternativos de regulação da floresta, com a inclusão de características ambientais e sociais para o manejo da paisagem. Os modelos foram construídos para uma fazenda modelo, com uma área de efetivo plantio de 3.49I ha, divididos em I 35 unidades de manejo. Os modelos de regulação foram formulados como modelo I, incluindo restrições de integridade para as unidades de gestão. Utilizou-se meta-heurística algoritmo genético para resolver os modelos. As propostas alternativas formuladas foram: (i) a formulação clássica de um modelo de regulação, (ii) a aplicação de restrições de adjacência e, (iii) a restrição das classes de idade por compartimento. Os modelos foram avaliados, economicamente, pelo valor presente líquido $(\mathrm{VPL})$ e, ambientalmente, pelo Índice de Área de Colheita (IAC). A aplicação da técnica de algoritmo genético foi eficiente para resolução dos modelos construídos com restrições de caráter sócioeconômico e ambiental. A imposição de restrições de adjacência reduziu o VPL global em cerca de $8 \%$, e a restrição de classes de idade por compartimento foi igual a $5 \%$, quando esta foi exigida no sétimo ano. A imposição das restrições de adjacência e por compartimento apresentaram uma melhoria na qualidade ambiental do modelo de regulação demonstrada pelo IAC.

\section{REGULATION OF EVEN-AGED FOREST WITH CONSTRAINT OF AGE CLASSES PER COMPARTMENT}

ABSTRACT: The objective of this study was to present the formulation of alternative models of regulation of the forest, with the inclusion of environmental and social characteristics for the management of the landscape. The models were constructed for a model farm, with an area of active planting of 349 I ha, divided into I 35 management units. The regulation models were formulated as type I model, including integrity constraints for the management units. We use meta-heuristic genetic algorithm for solving the models. The alternative proposals were formulated: (i) the formulation of a classical model of regulation, (ii) application of adjacency constraints, and (iii) the restriction of age classes per compartment. The models were evaluated economically by the net present value (NPV) and environmentally by the Crop Area Index (CAl). The application of the technique of genetic algorithm is efficient for solving the constructed models with socio-economic and environmental constraints. The imposition of restrictions adjacency reduced overall NPV by about $8 \%$, and the restriction of age classes per compartment was equal to $5 \%$ when this was required in seventh year. The imposition of adjacent and compartment restrictions showed an improvement in environmental quality of the regulatory model demonstrated by the CAl.

' Universidade Federal dos Vales do Jequitinhonha e Mucuri - Diamantina, MG

2 Universidade Federal de Viçosa - Viçosa, MG 


\section{INTRODUÇÃO}

Manejo florestal é a arte e a ciência da tomada de decisões, considerando a organização, uso e conservação da floresta e dos recursos relacionados, tendo como finalidade a produção de madeira, água, manutenção da fauna, recreação ou uma combinação desses fatores (BUONGIORNO; GILLESS, 2003). O processo de regulação florestal consiste em decidir quando, onde e quanto de madeira será cortada em uma determinada ordem para atingir os objetivos do manejo florestal (BOUNGIORNO; GILLESS, 2003; RODRIGUES, 200I). A programação matemática é utilizada na formulação e resolução de modelos de regulação florestal, auxiliando na obtenção da melhor solução para o problema em questão (BUONGIORNO; GILLESS, 2003; CHURCH; MURRAY, 1995; DAVIS et al., 2005).

Uma empresa florestal é constituída de um mosaico de talhões ou quadras de diferentes idades e tamanhos que são considerados como unidades de manejo. As unidades de manejo são, geralmente, agrupadas em compartimentos maiores (geralmente chamadas de regiões ou fazendas), decorrentes de fatores climáticos, fisiográficos, ambientais, sociais, culturais, administrativos e econômicos que influenciem na tomada de decisões da equipe de planejamento.

A incorporação de restrições que possibilitem uma maior fidelidade às características ambientais influenciadas pelos plantios florestais é uma realidade para as equipes de planejamento florestal nos dias atuais (DAVIS et al., 2005). Entretanto, a elaboração desses planos de manejo, requer uma interação de fatores ecológicos, espaciais, econômicos e sociais, resultando na consideração de uma quantidade de variáveis que inviabiliza a sua realização sem o auxilio de modernas ferramentas de planejamento (BUONGIORNO; GILLESS, 2003; DAVIS et al., 2005).

A utilização de características espaciais nos modelos de planejamento é essencial para a incorporação de variáveis ambientais como a manutenção da biodiversidade, a diminuição da erosão e assoreamento de rios e lagos, manutenção de corredores ecológicos, consideração de áreas de corte contínuas, ecologia de paisagem, zonas de amortização e distribuição regular de áreas de corte (BASKENT; JORDAN, 2002; BASKENT; KELES, 2005; CLEMENTS et al., 1990; NELSON; BRODIE, 1990).

A inclusão de restrições ambientais se dá, principalmente, pela imposição de restrições de adjacência, que consistem no controle das interações entre unidades de manejo da floresta, onde atividades de colheita em uma determinada unidade de manejo restringem ações de corte em unidades vizinhas ou adjacentes (MURRAY; $\mathrm{CHURCH}$, 1995). Essas restrições impedem que grandes áreas de corte contiguas sejam formadas. Existem diferentes formulações de restrições de adjacência, por meio de programação inteira mista (PIM), que se baseiam na formulação de pares entre os vizinhos ou em uma adaptação dessa formulação. A imposição dessas restrições implica em um maior esforço computacional, exigindo, às vezes, muitas horas, se não dias para a resolução de problemas com relativamente poucas unidades de manejo, por meio de algoritmos clássicos de programação inteira (MCDILL; BRAZE, 2000).

As técnicas de programação matemática são muito sensíveis ao número de variáveis de decisão, exibindo comportamento explosivo com a incorporação de relações espaciais existentes, causando um aumento exponencial no tempo de processamento (BASKENT, 200I). Técnicas heurísticas podem fornecer numerosas soluções factíveis com tempo e esforço computacional razoável (BARRETT; GILLESS, 2000; BASKENT; JORDAN, 2002; BETTINGER; SESSIONS, 2003; KURTTILA, 200I; MURRAY; SNYDER, 2000; NELSON et al., 199I; ÖHMAN, 200I). Os benefícios do uso dessas técnicas não envolvem somente a capacidade de solução de problemas de grande porte, mas também a simplicidade na formulação do problema (BASKENT; KELES, 2005). Dentre as metaheuristicas destacam-se: simulação de Monte Carlo, simulated anneling Busca tabu, e algoritmos genéticos. Esta última destaca-se pela facilidade de implementação e maior velocidade de processamento (LU; ERIKSSON, 2000; MULLER; BUTLER, 1997).

Objetivou-se, neste estudo, formular um modelo de regulação florestal considerando não somente a interação entre as unidades de manejo, mas, também, a distribuição das áreas de corte dentro de cada compartimento da floresta.

\section{MATERIAL E MÉTODOS}

\section{Dados}

Dados para um estudo de caso foram obtidos de uma fazenda modelo que representa uma situação encontrada em uma dada empresa florestal de Minas Gerais. A área total da fazenda modelo é de 9.750 ha, sendo que $35 \%$ (3.412 ha) de efetivo plantio e o restante da área constitui-se em reserva legal e áreas de preservação permanente (Figura I). A fazenda foi dividida em 135 unidades de manejo em função de características administrativas, edafoclimáticas e fisiográficas. A disposição das unidades de manejo e de classes de idade atual é apresentada na Figura I e 2. As unidades de manejo foram 
agrupadas em dois compartimentos com características distintas em função das unidades administrativas (Figura 3).

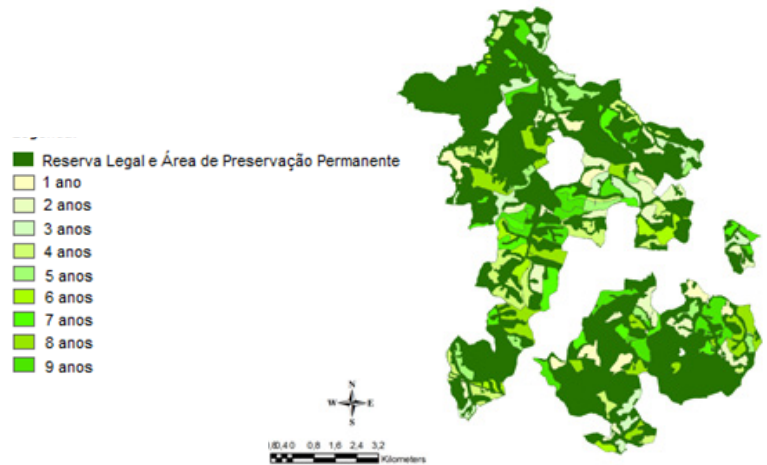

FIGURA I Distribuição espacial de classes de idade das áreas da fazenda modelo.

FIGURE I Spatial distribution of age classes in the areas of model farm.

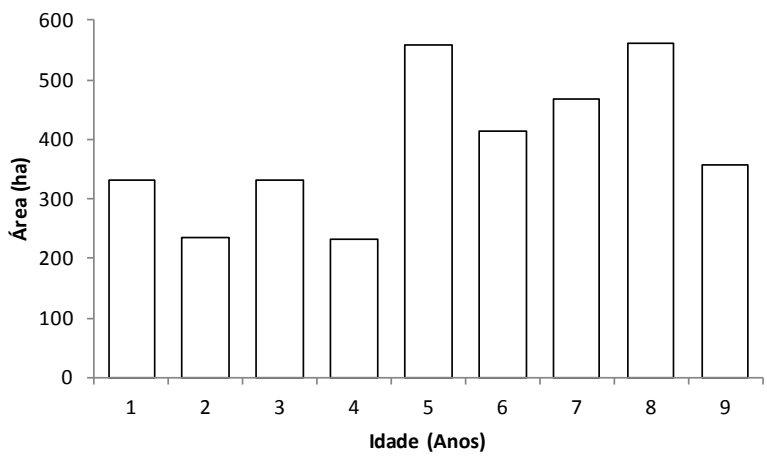

FIGURA 2 Distribuição inicial de classes de idade das áreas da fazenda modelo utilizada.

FIGURE 2 Initial distribution of age classes of the used farm model areas.

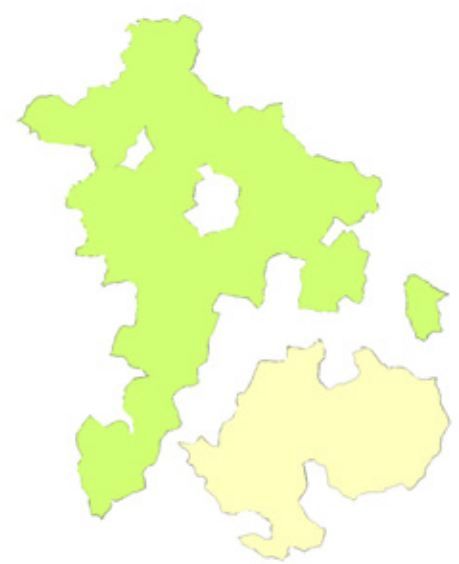

Legenda:

Regiâo A

Região $B$

FIGURA 3 Distribuição inicial de classes de idade das áreas da fazenda modelo utilizada.

FIGURE 3 Initial distribution of age classes of the used farm model areas.

\section{Modelos matemáticos}

O modelo de regulação foi formulado empregando planilhas eletrônicas do software Microsoft Excel e código em Visual Basic for Applications. A abordagem utilizada para a geração de unidades de manejo foi o modelo I descrito em Johnson e Scheurman (1977), com a utilização de variáveis binárias para as variáveis decisórias, resultando na função objetivo $I$, sujeita às equações $2,3,4,5$ e 6 , onde $Z=$ Valor Presente Líquido Global $(\mathrm{R} \$) ; \mathrm{x}_{\mathrm{ij}}=$ variáveis de decisão, representando a j-ésima alternativa de manejo adotada na i-ésima unidade de manejo; $c_{i j}=$ Valor Presente Líquido de cada unidade de manejo $\mathrm{i}$, manejada segundo a alternativa de manejo j; $\mathrm{m}=$ número total de unidades de manejo; $\mathrm{n}=$ número total de alternativas de manejo na i-ésima unidade de manejo; $V_{\text {iik }}=$ volume $\left(\mathrm{m}^{3}\right)$ produzido pela i-ésima unidade de manejo quando a j-ésima alternativa de manejo é adotada, para o período

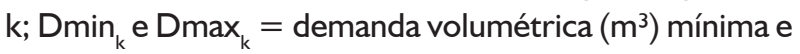
máxima respectivamente, em cada período do horizonte de planejamento; $A_{l k}$ representa a área do povoamento na idade I e período k; e IR idade regulatória: Função Objetivo:

MAXZ $=\sum_{i=1}^{m} \sum_{j=1}^{n} c_{i j} x_{i j}$

\section{Sujeito a:}

$\sum_{j=1}^{n} x_{i j}=I \quad \forall i$

$\sum_{i=1}^{m} \sum_{j=1}^{n} V_{i j} x_{i j} \geq D \min _{k} \quad\{k=0, I, \ldots, H-l\}$

$\sum_{i=1}^{m} \sum_{j=1}^{n} V_{i j} x_{i j} \leq \operatorname{Dmax}_{k} \quad\{k=0, I, \ldots, H-I\}$

$\sum_{l=1}^{\mathbb{R}} A_{l k}=\left(\frac{\text { Áreatotal }}{\mathbb{I R}}\right) \quad\{k=H-I\}$

$x_{i j} \in\{0, I\}$

De acordo com esse modelo de programação inteira mista, a maximização do valor presente global [I] está sujeita às restrições de singularidade [2] e [6] e às produções mínima [3] e máxima [4], em cada período do horizonte de planejamento. A restrição [5] garante o estabelecimento da regulação florestal. Definiu-se o 
horizonte de planejamento como de I,5 ciclos, conforme sugerido por Leuschener (1984), sendo a rotação de 6 anos, considerando nesse caso um ciclo de duas rotações, o horizonte de planejamento foi de 18 anos.

As intervenções nos povoamentos incluíram corte seguido de imediata reforma ou corte com reforma no próximo período, o que significa que apenas um regime de manejo (alto-fuste) foi avaliado. Possibilitou-se a variação da idade de corte entre 5 a 9 anos, dentro do horizonte de planejamento. Utilizou-se como idade regulatória a idade de 6 anos. Para fins de comparação, obteve-se o mesmo modelo sem a inclusão das restrições de adjacência e restrições de classes de idade por compartimento.

\section{Restrição de classes de idade por compartimento}

A formulação dessa restrição 7 constituiu na exigência de áreas em classes de idade iguais em cada compartimento da floresta. $O$ inicio da aplicação da restrição ocorreu em diferentes anos do horizonte de planejamento, variando entre o sétimo e o último ano do horizonte de planejamento. $A_{\mathrm{lk}}$ representa as áreas de cada unidade de manejo i na idade l e período k; e $\mathrm{At}_{\mathrm{q}}$ representa a área total do compartimento q.

$$
\sum_{i=1}^{n} A_{i k}=\left(\frac{A t_{q}}{\sum_{l=1}^{n} l}\right) \quad\{k=7,8, \ldots . H-l\}
$$

\section{Restrição de adjacência}

Em razão da topografia acidentada da área de estudo, as unidades de manejo foram consideradas adjacentes quando a distância entre seus limites (bordas) apresentava-se menor ou igual a $50 \mathrm{~m}$. A restrição de adjacência 8 para evitar o corte de unidades de manejo adjacentes encontra-se abaixo, onde $n_{i}$ refere-se ao número de unidades de manejo adjacentes que apresentam cortes no período k (MCDILL; BRAZE, 2000).

$$
n_{i} x_{i j}+\sum_{i \in N_{i}} x_{i j} \leq n_{i} \quad \forall i, k
$$

\section{Algoritmo genético}

O AG foi implementado em uma rotina computacional, utilizando o ambiente de programação Visual Basic for Applications em conjunto com o software MS Excel. As soluções (indivíduos) geradas para o problema em questão apresentaram o formato de um vetor $\mathrm{V}(\mathrm{x})=\left\{\mathrm{X}_{11}, \mathrm{X}_{12}, \ldots, \mathrm{X}_{\mathrm{ij}}\right\}$, onde a variável de decisão $X_{i j}\left(X_{i j} \in\{0, \mid\}\right)$,simboliza a alternativa de manejo $\mathrm{j}(\mathrm{j}=\mathrm{I}, 2, \ldots, \mathrm{n})$ assinalada à unidade de manejo $\mathrm{i}(\mathrm{i}$ $=$ I,2...m) (RODRIGUES et al., 2004).

A função de aptidão do algoritmo genético empregada 9 baseou-se na aplicação de penalidades a função objetivo, que consiste em maximizar o valor presente líquido global (VPL). As penalidades impostas referem-se à variação volumétrica acima ou abaixo da demanda proposta, áreas com cortes adjacentes e, distribuição de classes de idades irregular ao final do horizonte de planejamento. Na função fitness $Z$ = valor da função de aptidão; $x_{i j}=$ variáveis de decisão, representando a j-ésima alternativa de manejo adotada na i-ésima unidade de manejo; $c_{i j}=$ valor presente líquido de cada unidade de manejo $i$, manejada segundo $\mathrm{a}$ alternativa de manejo j; $\mathrm{m}=$ número total de unidades de manejo; $\mathrm{n}=$ número total de alternativas de manejo na i-ésima unidade de manejo; $\mathrm{V}_{\mathrm{ijk}}=$ volume $\left(\mathrm{m}^{3}\right)$ produzido pela i-ésima unidade de manejo quando a j-ésima alternativa de manejo é adotada, para o período $\mathrm{k} ; \mathrm{H}_{\mathrm{k}}=$ demanda volumétrica $\left(\mathrm{m}^{3}\right)$, em cada período do horizonte de planejamento; $\alpha, \beta$ e $\gamma$ são os coeficientes de penalidade associados a cada restrição; $A_{\mathrm{lkq}}$ refere-se a áreas do compartimento $q$ cortadas no período $k$; $A_{1}$ $=$ área regulatória e $A_{i}=$ área da unidade de manejo $i$, como mostrado a seguir:

$$
\begin{aligned}
& M a x=\sum_{i=1}^{m} \sum_{j=1}^{n} c_{i j} x_{i j}-\propto\left(H_{k}-\sum_{i=1}^{m} \sum_{j=1}^{n} V_{i j} x_{i j}\right)- \\
& -\beta\left(A_{l}-\sum_{q=1}^{2} \sum_{k=1}^{H-1} \sum_{l=1}^{n} A_{l k q}\right)^{2}-\gamma\left(A_{l}-\sum_{i=1}^{m} A_{i} x_{i}\right)^{2}
\end{aligned}
$$

A população inicial do $A G$ foi constituída de 30 indivíduos gerados aleatoriamente, considerando a viabilidade de cada solução pela restrição de singularidade. Utilizou-se o crossover de múltiplos pontos e uma taxa de mutação de $0,6 \%$ para cada indivíduo da população. $A$ seleção de indivíduos ocorreu por elitização. A parada do processo iterativo corrida do algoritmo ocorreu, quando, após a estabilização do fitness, o AG realizou 20 novas gerações.

\section{Produção, receita e custo}

Considerou-se somente o plantio de um clone híbrido (Eucalyptus urophylla x Eucalyptus grandis), como sendo a única fonte de madeira utilizada pela empresa. $A$ 
curva de produção 10 obtida a partir de dados de parcelas de inventário florestal, onde a taxa de juros utilizada foi de $8,75 \%$ ao ano e o valor de venda da madeira foi de $\mathrm{R} \$$ $80,00 / \mathrm{m}^{3}$. Os valores de custo das atividades florestais utilizados foram obtidos em Binoti (2010).

Volume $=\frac{197,833}{I+75,674 e^{-1,315 / \text { dade }}}$

\section{Avaliação da dispersão das intervenções nas unidades de manejo}

O efeito da restrição de adjacência foi avaliado pela média ponderada do inverso do quadrado da menor distância entre unidades de manejo cortadas no mesmo período, II pelo quadrado das áreas das unidades de manejo que sofrem intervenção. No índice utilizado IAC = índice de área de colheita, $A_{1}=$ área da unidade de manejo i com intervenção, em hectares, $d_{i}=$ distância até a unidade de manejo mais próxima com intervenção, em quilômetros.

$$
I A C=\frac{\sum_{k=0}^{H-1} \sum_{i=1}^{m} A_{i}^{2}\left(\frac{1}{d_{i}}\right)^{2}}{\sum_{i=1}^{m} A_{i}}
$$

\section{RESULTADOS E DISCUSSÃO}

A formulação do modelo de planejamento resultou em um modelo com 8.755 variáveis decisórias. As formulações das restrições de adjacência resultaram em 37.555 restrições. As restrições formuladas via classes de idade por compartimento resultaram em 66 restrições quando a aplicação ocorre no sétimo ano e 6 restrições com a aplicação no último ano do horizonte. $\bigcirc$ tempo de processamento para o modelo com restrição de adjacência em relação aos modelos com as restrições formuladas por classes de idade e compartimento aumentou em cerca de $50 \%$, sendo que não houve diferença na resolução entre as diferentes formas de aplicação das restrições por compartimento, contudo, não foi objetivo deste trabalho avaliar o tempo de processo para a resolução dos modelos.

A variação da produção volumétrica dos modelos de regulação é apresentada na Figura 4. A imposição das restrições de adjacência reduziu o VPL global, em torno de $8 \%$, sendo que a redução do VPL global pela exigência de classes de idade iguais foi de $5 \%$ no sétimo ano apresenta um aumento gradual até o último ano como apresentado na Figura 4. O valor da função objetivo para os modelos com e sem a inclusão das restrições de adjacência foi de $\mathrm{R} \$$ 17.|43.857, I8 e R\$ 18.599.169,38, respectivamente, e de $R \$$ 17.523. I54,00 para o sétimo ano com a exigência de classes de idade constante.

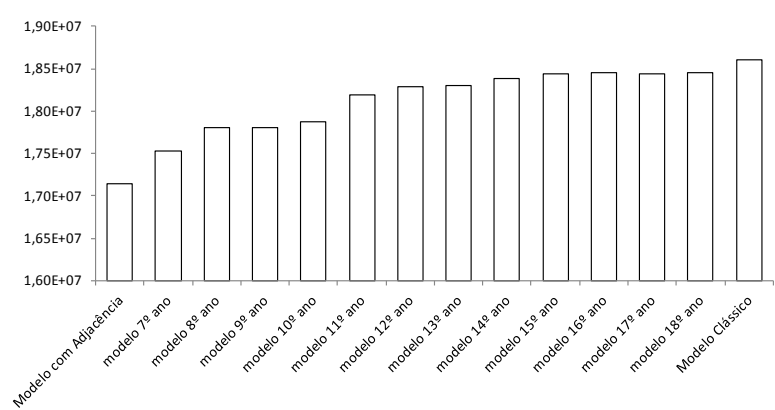

FIGURA 4 VPL global durante todo o horizonte de planejamento para cada modelo.

FIGURE 4 NPV overall throughout the planning horizon for each model.

Os valores do IAC para os modelos com e sem a inclusão da restrição de adjacência foram de 0,246436 e 0,615032, respectivamente. A dinâmica do índice com relação ao ano de imposição das restrições de classes de idade apresentou uma tendência de redução do índice com o passar dos anos, sendo o menor valor de 0,4 I 37034 obtido no oitavo ano. A variação do IAC apresenta tendência de aumento com o passar dos anos onde a restrição e implementada. $A$ variação do IAC é apresentada na Figura 5.

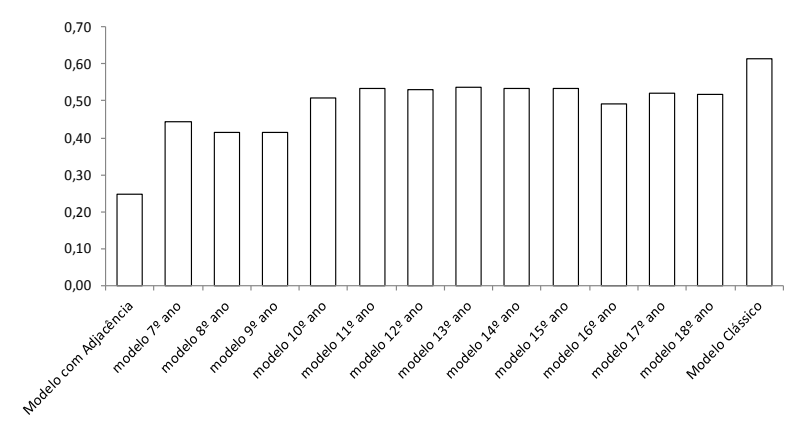

FIGURA 5 Variação do IAC em função de diferentes imposições formas de imposição da restrição de classes de idade por compartimento.

FIGURE 5 Variation of the IAC for different ways of imposing the restriction for age class per compartiment.

A aplicação de restrições espaciais influencia negativamente no valor da função objetivo. Daust e Nelson (1993), encontraram reduções de até $29 \%$ do volume produzido com a aplicação de restrições de adjacência 
(green-up), quando comparadas com a solução típica de programação linear. Yoshimoto e Brodie (1994) obtiveram uma redução de $40 \%$ do VPL com a aplicação desse mesmo tipo de restrição. Castro (2007) observou uma redução de cerca de $6 \%$ para o valor de VPL global, em um trabalho para avaliação da imposição de restrições de adjacência. Neste estudo, obteve-se uma redução de $8 \%$ com a aplicação da restrição de adjacência clássica, a imposição das restrições de distribuição de idades por compartimento reduziu em $5 \%$ o valor de VPL global, sendo que o efeito tende a aumentar com a imposição da restrição em anos posteriores do horizonte de planejamento.

Pela avaliação do IAC a implementação das restrições apresenta um efeito benéfico para o manejo da paisagem, sendo que a imposição da restrição em anos mais próximos do inicio do horizonte, sendo que o beneficio ambiental apresenta tendência de redução com a imposição em anos mais avançados do horizonte de planejamento.

\section{CONCLUSÕES}

A metodologia proposta apresenta efeito benéfico para o manejo da paisagem podendo ser utilizada em modelos de planejamento florestal.

\section{REFERÊNCIAS}

BARRETT, T. M.; GILLESS, J. K. Even-aged restrictions with subgraph adjacency. Annals Operation Research, New York, v. 95, p. I59-I75, 2000.

BASKENT, E. Z. Combinatorial optimization in forest ecosystem management modeling. Turkish Journal of Agriculture \& Forestry, Bakanliklar, v. 25, p. 187-194, 200 I.

BASKENT, E. Z.; JORDAN, G. A. Forest landscape management modelling using simulated annealing. Forest Ecology and Management, Philadelphia, v. I65, p. 2945, 2002.

BASKENT, E. Z.; KELES, S. Spatial forest planning: a review. Ecological Modelling, Philadelphia, v. 188, p. I45-173, 2005.

BETTINGER, P.; SESSIONS, J. Spatial forest planning: to adopt, or not to adopt? Journal of Forestry, Bethesda, v. I0I, n. 2, p. 24-29, 2003.

BINOTI, D. H. B. Estratégias de regulação de florestas equiâneas com vistas ao manejo da paisagem. 20I0. I57 f. Dissertação (Mestrado em Ciência Florestal) - Universidade Federal de Viçosa, Viçosa, 2010.

BUONGIORNO, J.; GILLES, J. K. Decision methods for forest resource management. San Diego: Academic, 2003. 439 p.
CASTRO, R. R. Regulação de florestas equiâneas incluindo restrições de adjacência. 2007. 64 f. Dissertação (Mestrado em Ciência Florestal) - Universidade Federal de Viçosa, Viçosa, 2007.

CHURCH, R.; MURRAY, A. Heuristic solution approaches to operational forest planning problems. Operation Research Spektrum, Heidelberg, v. 17, p. 193-203, 1995.

CLEMENTS, S. E.; DALLEIN, P. L.; JAMNICK, M. S. An operational spatially constrained harvest scheduling model. Canadian Journal of Forest Research, Ottawa, v. 20, p. |438-|447, 1990.

DAUST, D. K.; NELSON, J. D. Spatial reduction factors for strata-based harvest schedules. Forest Science, Bethesda, v. 39, n. I, p. 152-165, 1993.

DAVIS, L. S.; JOHNSON, K. N.; BETTINGER, P.; HOWARD, T. E. Forest Management: to sustain ecological, economic, and social values. Columbus: McGraw-Hill, 2005. 804 p.

JOHNSON, K. N.; SCHEURMAN, H. L. Techniques for prescribing optimal timber harvest and investment under different objectives discussion and synthesis. Bethesda: Forest Science, 1977. (Forest Science Monograph, 18).

KURTTILA, M. The spatial structure of forests in the optimization calculations of forest planning: a landscape ecological perspective. Forest Ecology and Management, Philadelphia, v. I42, p. 129142, 200 I.

LEUSCHNER, W. A. Introduction to forest resource management. New York: J. Wiley, 1984. 284 p.

LU, F; ERIKSSON, K. O. Formation of harvest units with genetic algorithms. Forest Ecology and Management, Philadelphia, v. I30, p. 57-67, 2000

MCDILL, M. E.; BRAZE, J. Comparing adjacency constraint formulations for randomly generated forest planning problems with four age-class distributions. Forest Science, Bethesda, v. 46, p. 423-436, 2000.

MULLEN, D. S.; BUTLER, R. M. The design of a genetic algorithm based spatially constrained timber harvest scheduling model. Disponível em: <http://www.for.msu. edu/e4/e4ssafr97.html>. Acesso em: 10 dez. 1997.

MURRAY, A. T.; CHURCH, R. Measuring the efficacy of adjacency constraint structure in forest planning models. Canadian Journal of Forest Research, Ottawa, v. 25, p. |4|6-|424, 1995.

MURRAY, A. T.; SNYDER, S. Spatial modeling in forest management and natural resource planning. Forest Science, Bethesda, v. 46, n. 2, p. I53-I56, 2000. 
NELSON, J.; BRODIE, J. D. Comparison of random search algorithm and mixed integer programming for solving area-based forest plans. Canadian Journal of Forest Research, Ottawa, v. 20, p. 934-942, 1990.

NELSON, J.; BRODIE, J. D.; SESSIONS, J. Integrating shortterm, area-based logging plans with long-term harvest schedules. Forest Science, Bethesda, v. 37, n. I, p. I0I122, 1991 .

ÖHMAN, K. Forest planning with consideration to spatial relationships. 200I. 32 p. Thesis (Doctoral in Forest Resource Management and Geomatics) - Universitatis Agriculturae Sueciae, Silvestria, 200 I.
RODRIGUES, F. L. Metaheurística e sistema de suporte à decisão no gerenciamento de recursos florestais. 200I. 225 f. Tese (Doutorado em Ciência Florestal) Universidade Federal de Viçosa, Vicosa, 200I.

RODRIGUES, F. L.; LEITE, H. G.; SANTOS, H. N.; SOUZA, A. L.; SILVA, G. F. Metaheuristica algoritmo genetic para solução de problemas de planejamento florestal com restrições de integridade. Revista Árvore, Viçosa, v. 22, n. 2, p. 233-245, 2004.

YOSHIMOTO, A.; BRODIE, J. D.; SESSIONS, J. Comparative analysis of algorithms to generate adjacency constraints. Canadian Journal of Forest Research, Ottawa, v. 24, p. I277-I288, 1994. 